\title{
Design studies of a compact superconducting rf crab cavity for future colliders using $\mathrm{Nb} / \mathrm{Cu}$ technology
}

\author{
K. Papke, ${ }^{*}$ A. A. Carvalho, C. Zanoni, ${ }^{\dagger}$ and A. Grudiev \\ CERN, CH-1211 Geneva 23, Switzerland
}

(Received 26 March 2018; revised manuscript received 14 May 2019; published 3 July 2019)

\begin{abstract}
The design of a compact crab cavity using $\mathrm{Nb} / \mathrm{Cu}$ technology is presented. The cavity shape is based on a ridged waveguide resonator with wide-open apertures to provide access to the inner surface of the cavity and facilitate coating. It also provides natural damping for higher-order modes (HOMs) and comparatively low longitudinal and transverse impedances. A first prototype is being fabricated, originally within the HLLHC framework and now for the Future Circular Collider study. The optimized shape is characterized and compared with respect to peak surface field balance, multipolar momentum components, and longitudinal and transverse impedances. Further investigations include the identification of multipacting barriers, the frequency sensitivity to pressure fluctuations in the helium bath, the radio frequency (rf) design of a fundamental mode coupler, and considerations of HOM damping. Along these topics, several methods have been established serving a wider range of applications, in particular, for the rf loss evaluation, the postprocessing of multipacting simulations, and a detailed error analysis of the pressure sensitivity simulations.
\end{abstract}

DOI: 10.1103/PhysRevAccelBeams.22.072001

\section{INTRODUCTION}

Several studies for future accelerators include now the use of superconducting crab cavities, which are rf deflecting type cavities operating at zero-crossing and tilting the particle bunches prior to the collision point in order to provide head-on collisions. The crab crossing concept proposed by Palmer [1] allows for collisions with a maximum number of particles in each bunch and has been successfully tested at KEK for the KEKB electron-positron collider [2]. Proof-of-principles for Hadron machines are currently under development at CERN in the frame of the HL-LHC and as a concept for the Future Circular Collider (FCC). For the HL-LHC, various compact designs of superconducting deflecting type cavities have been proposed in which "compact" relates to the fact that the transverse cavity dimension is significantly smaller than half the wavelength. Consequently, pillbox or elliptical cavities operating in $\mathrm{TM}_{110}$-like mode are excluded from the very beginning. This requirement follows from the LHC specifications of a rf frequency equal to $400 \mathrm{MHz}$ while the distance between the two beam pipes at the foreseen cavity

\footnotetext{
*kai.papke@cern.ch

Present address: European Southern Observatory (ESO), Munich, Germany.

Published by the American Physical Society under the terms of the Creative Commons Attribution 4.0 International license. Further distribution of this work must maintain attribution to the author(s) and the published article's title, journal citation, and DOI.
}

locations is about $194 \mathrm{~mm}$ [3]. Three designs have been evaluated, fabricated, and rf tested so far: (i) The double quarter wave crab cavity (DQWCC) developed at BNL in Upton, New York, USA [4,5], which will be used for the above mentioned proof-of-principle test [6], (ii) the rf dipole crab cavity (RFDCC) developed in collaboration between the Old Dominion University (ODU) in Norfolk, Virginia, USA and SLAC National Laboratory in Menlo Park, California, USA [7], and (iii) the four-rod UK crab cavity (4RCC) developed at the Lancaster University in Bailrigg, Lancaster, UK in collaboration with the Cockcroft Institute in Daresbury, Warrington, UK [8].

The cavity discussed in this work is denoted wide open waveguide crab cavity (WOWCC), was originally conceived within the HL-LHC development [9] and is now part of the FCC study [10]. The goal, at this stage, is to increase the readiness level of the technology in a likely scenario, therefore the HL-LHC constraints are applied. The use of the HL-LHC specifications has the advantage of facilitating a future benchmarking with the other crab cavity designs. In the following, parameters such as LHC $400 \mathrm{MHz}$ rf frequency, beam pipe radius, and distance are therefore used.

The DQWCC, the RFDCC, and the $4 \mathrm{RCC}$ are made in 3-4 $\mathrm{mm} \mathrm{Nb}$ sheets. The WOWCC is instead a $\mathrm{Nb}$ coating on $\mathrm{Cu}$ substrate, an approach supported by several arguments. The high thermal conductivity of $\mathrm{Cu}$ not only provides very good cooling of the superconducting $\mathrm{Nb}$ thin film but also anticipates thermal quenching. In addition, $\mathrm{Cu}$ allows for very precise cavity shapes using modern CNC five-axis milling machines. Typically, their accuracy 
is by at least one order of magnitude higher than the accuracy achieved by bulk Nb sheet techniques. Moreover, Lorentz force detuning is less problematic in case of $\mathrm{Nb}$ coated cavities if the substrate is much thicker than the common $\mathrm{Nb}$ sheets. The outer cavity shape can be designed such that the impact of pressure fluctuations within the helium bath on the frequency vanishes meaning that the cavity wall itself serves as a passive tuner. Other advantages of coated cavities in comparison to conventional bulk $\mathrm{Nb}$ cavities are the significant lower material costs and the empirically much lower sensitivity to the earth magnetic field of $\mathrm{Nb}$ film cavities [11]. In contrast, solid bulk $\mathrm{Nb}$ cavities typically show lower cryogenic loss and are more easily handled during fabrication and installation. The list of advantages and disadvantages of the different technologies is by no means complete, but it serves well in motivating $\mathrm{R} \& \mathrm{D}$ for a $\mathrm{Nb} / \mathrm{Cu}$ crab cavity concept.

The WOWCC is based on a ridged waveguide resonator with wide open apertures to allow direct access to the interior for the surface preparation and coating [Fig. 1(a)]. Due to the large apertures, the number of trapped higherorder modes (HOMs) is comparably low which eventually facilitates their damping. Likewise, the longitudinal and transverse impedances are relatively low. The same approach was applied to the so-called quasiwaveguide

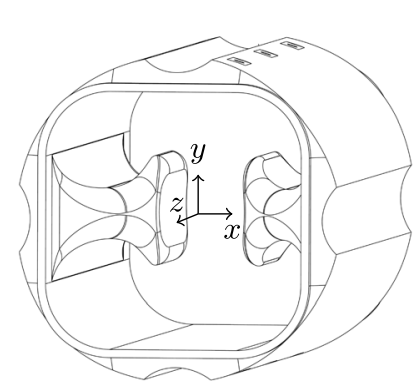

(a)

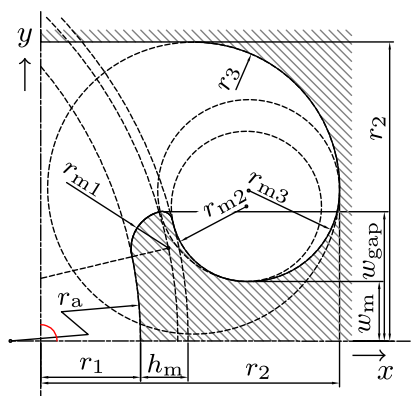

(b)

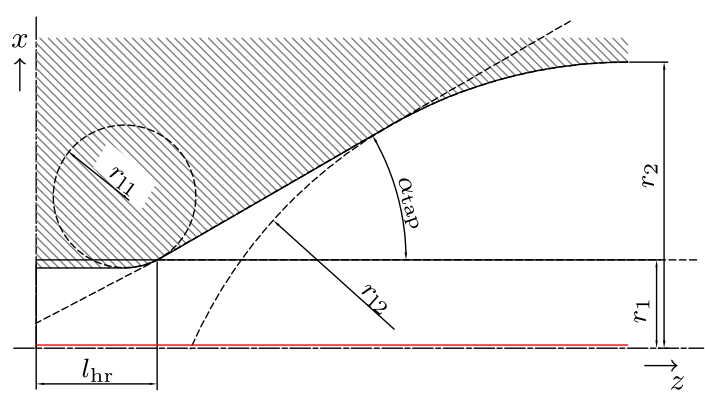

(c)

FIG. 1. Design of the wide-open-waveguide crab cavity (WOWCC). (a) Perspective view of the finalized cavity shape. Bunches are deflected in the horizontal direction by the electric field created between the two mushroom-shaped ridges. A quarter of the transverse and longitudinal cross section with the various design parameters are shown in (b) and (c), respectively. The location of the beam is highlighted in red. multicell resonator (QMiR) for the reason of impedance reduction at the Advanced Photon Source's short pulse $\mathrm{x}$-ray project [12]. Furthermore, in the WOWCC, the transitions from the central part with two ridges to the beam pipe part with no ridges have smooth tapers of $30 \mathrm{deg}$ in order to facilitate the coating of these very critical regions. This helps to reduce further its longitudinal and transverse impedances.

In this paper, we describe the design of the first prototype, which addresses the feasibility issues of fabrication and coating of the cavity itself with the final goal of measuring the rf power loss at $4.5 \mathrm{~K}$ as a function of the cavity deflecting voltage. After a general overview of the shape optimization in Sec. II, the cavity is further characterized by means of detailed power loss and quality factor calculations in Sec. III. Different methods are presented accounting for the field dependence of the surface resistance. In Sec. IV, the longitudinal and transverse impedances are analyzed. Potential dangerous HOMs are identified and addressed by appropriate damping approaches. Furthermore, the rf design of the fundamental mode coupler is discussed in Sec. V followed by multipacting analyses of the bare cavity and various evaluations of the frequency sensitivity against pressure fluctuations in the helium bath in Secs. VI and VII, respectively. For the latter one, different numerical techniques are benchmarked, serving as a precious reference for future cavity developments.

\section{CAVITY DESIGN}

The shape of the internal cavity surface is directly related to both the rf performance and the $\mathrm{Nb}$ coating process. The existing designs of LHC crab cavities are very difficult if not impossible to coat with $\mathrm{Nb}$ since they provide only limited access either through the beam pipe ports with a diameter of $84 \mathrm{~mm}$ or through even smaller coupler ports. To facilitate the access for sputtering cathodes, the cavity design presented in this paper is based on a piece of a double-ridged waveguide with apertures left open at both ends (Fig. 1). The reentrant transverse ridge profile, sketched in Fig. 1(b), allows for a very compact transverse cavity dimension. A setup of five sputtering cathodes is considered: One in the cavity center along the beam line and a further cathode accounting for each reentrant profile (approximately in the center of the circle with the radius $\left.r_{\mathrm{m} 3}\right)$. Figures 2(a) and 2(b) show a quarter of the transverse cross section at the cavity center together with the electric and magnetic field distributions, respectively, obtained by a rf eigenmode simulation using COMSOL [13]. In analogy to the eigenmode solutions of a pillbox, the mode can be denoted as $\mathrm{TE}_{111}$-like mode. The particle deflection is caused by the transverse electric field between the two mushroom-shaped ridges while the magnetic field provides a small parasitic contribution to the overall transverse net kick [7]. The following paragraphs briefly discuss all 


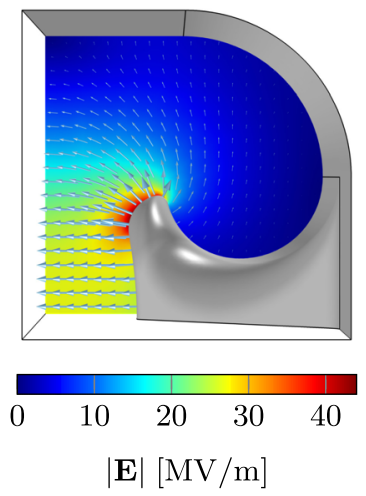

(a)

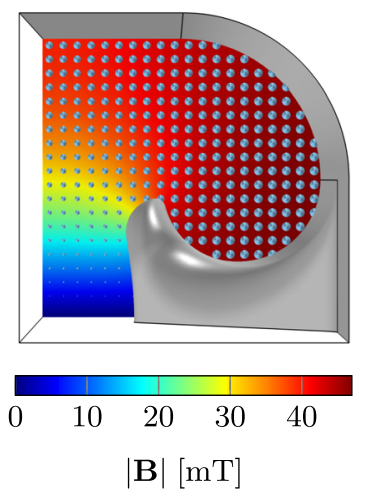

(b)

FIG. 2. Quarter of the transverse cross section at the cavity center. The electric (a) and magnetic (b) fields of the $\mathrm{TE}_{111}$-like fundamental mode are scaled to the nominal deflecting voltage of $V_{\perp, 0}=3 \mathrm{MV}$.

design parameters taken into account for the interior shape optimization.

Let the z-axis coincide with the longitudinal axis of the cavity, the transverse voltage is calculated according to the integrated Lorentz force along the center axis as follows:

$$
V_{\perp}=\int_{-\infty}^{\infty}\left(E_{\perp}-\beta Z_{0} H_{\perp}\right) \exp \left(j \frac{\omega z}{\beta c_{0}}\right) d z
$$

with the impedance of free space $Z_{0}$, the angular frequency $\omega=2 \pi f$, and $\beta$, the ratio of the particle velocity to the speed of light $c_{0}$. Here, the components of the transverse electric and magnetic field intensities, $E_{\perp}$ and $H_{\perp}$, are chosen such that their Poynting vector is oriented in the positive $z$-direction. Since dipole modes provide a deflecting voltage inversely proportional to the squared distance between the two ridges [[14], pp. 504-508], the parameter $r_{1}$ is chosen to be as small as possible and limited by the LHC beam pipe radius to $42 \mathrm{~mm}$. Moreover, Eq. (1) contains implicitly the transit time factor. Thus, for a given frequency, the cavity length notably influences the deflecting voltage. It was found that the tapering angle $\alpha_{\text {tap }}$ shown in Fig. 1(c) has a minor impact in comparison to the elongation of the mushroom profile, $l_{\mathrm{hr}}$.

A further important aspect in optimizing superconducting cavities are the peak surface electric and magnetic fields, $E_{p k}$ and $B_{p k}$ limited by field emission and excessive rf losses, respectively. Comparing Figs. 2 and 3, neither the electric nor magnetic peak surface field is located at the cavity center but rather at the beginning of the transition toward the beam pipe. A transition with maximized curvature radii avoiding this field enhancement constitutes the main problem in the cavity design, in particular, because the design tools available in CST [15], HFSS [16], or COMSOL such as fillets with constant curvature radius or lofts are not sufficient to face this problem. Fillets

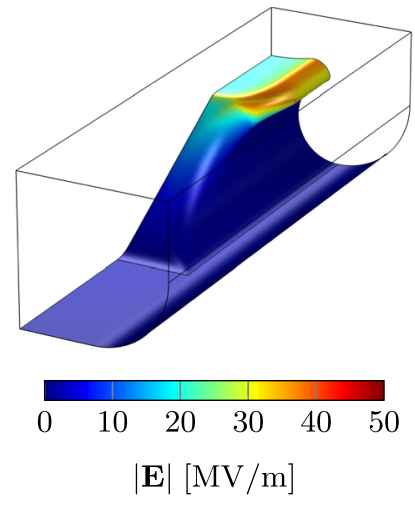

(a)

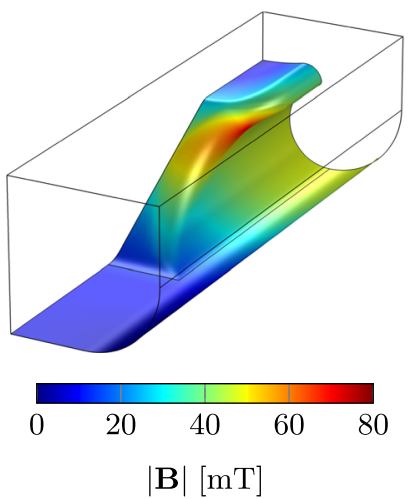

(b)
FIG. 3. Eighth of the inner cavity surface. The electric (a) and magnetic (b) field distributions of the $\mathrm{TE}_{111}$-like fundamental mode is scaled to the nominal deflecting voltage of $V_{\perp, 0}=3 \mathrm{MV}$.

with variable curvature radius using CATIA [17] notably improve the situation by reducing the peak fields down to $45 \mathrm{MV} / \mathrm{m}$ and $78 \mathrm{mT}$. The peak magnetic field may still be challenging for $\mathrm{Nb} / \mathrm{Cu}$ technology at $4.5 \mathrm{~K}$ and at $400 \mathrm{MHz}$. Recently reported results [18] of various such coated cavities operating at $100 \mathrm{MHz}, 400 \mathrm{MHz}$, and $1.5 \mathrm{GHz}$ at temperatures of $2 \mathrm{~K}$ and $4.5 \mathrm{~K}$ provide certain evidence of a stable superconducting state. However, since this conclusion is based on comparing and extrapolating the measured surface resistances versus peak magnetic field of different cavities, ultimately, only a rf cold test of this particular cavity prototype will explore realistic performances and acceptable peak surface fields. Note, the stability of the superconducting state is strongly related to the thermal stability of the cavity, which has been studied in [9].

The shape of the capacitive plates defined by the parameters $w_{\text {gap }}, r_{\mathrm{a}}$, and $r_{\mathrm{m} 1}$ is optimized both, to reduce the surface electric field and to improve the deflecting field quality by minimizing its sextupolar component $b_{3}$. Using cylindrical coordinated $(r, \varphi, z)$, multipolar components $[19,20]$ can be evaluated according to:

$$
b_{n}=\frac{j n}{\omega} \frac{1}{\pi} \int_{-\pi}^{\pi} \frac{\cos (n \varphi)}{r^{n}} \int_{0}^{L} E_{z} \exp \left(j \frac{\omega z}{\beta c_{0}}\right) d z d \varphi .
$$

The HL-LHC specification constrains the sextupolar component to $\left|b_{3}\right| \leq 1.5 \mathrm{Tm} / \mathrm{m}^{2}$ at $10 \mathrm{MV}$ deflecting voltage [21].

As previously mentioned, the operating frequency needs to be $400 \mathrm{MHz}$ [3] and is adjusted via the cavity radius $r_{2}$. However, the distance of $194 \mathrm{~mm}$ between both beam pipes asks for a cavity radius to be smaller than $144 \mathrm{~mm}$ including the cavity wall. It is worth noting that this restriction applies only in the plane of both beam pipes. The mechanical design of the first prototype provides 
TABLE I. Main rf parameters of various rf crab cavities.

\begin{tabular}{lcccccc}
\hline \hline Parameter & Unit & WOWCC & DQWCC [5] & RFDCC [22] & 4RCC [8] & QMiR [12] \\
\hline cross section $(W \times H)$ & {$[\mathrm{mm}]$} & $250 \times 250$ & $321 \times 278$ & $281 \times 281$ & $120 \times 145$ & $41.9 \times 41.9$ \\
cavity length & {$[\mathrm{mm}]$} & $1000^{\mathrm{a}}$ & 660 & 950 & 501 & $300^{\mathrm{a}}$ \\
smallest aperture radius & {$[\mathrm{mm}]$} & 42 & 42 & 42 & 42 & 6 \\
design frequency & {$[\mathrm{MHz}]$} & 400 & 400 & 400 & 400 & 2815 \\
geometry factor $\mathrm{G}$ & {$[\Omega]$} & 108.9 & 87.0 & 107.0 & 62.1 & 130.0 \\
deflecting voltage $V_{\perp, 0}$ & {$[\mathrm{MV}]$} & 3.0 & 3.4 & 3.4 & 3.0 & 2.0 \\
$(R / Q)_{\perp}$ (circuit definition) & {$[\Omega]$} & 171.8 & 214.7 & 215.0 & 269.1 & 520.0 \\
$E_{p k}$ at $V_{\perp, 0}$ & {$[\mathrm{MV} / \mathrm{m}]$} & 45.3 & 37.6 & 33.0 & 30.7 & 54.0 \\
$B_{p k}$ at $V_{\perp, 0}$ & {$[\mathrm{mT}]$} & 78.3 & 72.8 & 56.0 & 54.9 & 75.0 \\
helium bath temperature & {$[\mathrm{K}]$} & 4.5 & 2.0 & 2.0 & 2.0 & 2.0 \\
$\left\|b_{3}\right\|$ at $V_{\perp}=10 \mathrm{MV}$ & {$\left[\mathrm{Tm} / \mathrm{m}^{2}\right]$} & $9.0 \times 10^{-2}$ & 1.5 & $4.8 \times 10^{-1}$ & $9.0 \times 10^{-1}$ & $2.7 \times 10^{-2}$ \\
$\left\|b_{5}\right\|$ at $V_{\perp}=10 \mathrm{MV}$ & {$\left[\mathrm{Tm} / \mathrm{m}^{4}\right]$} & $1.4 \times 10^{3}$ & $1.7 \times 10^{5}$ & $2.2 \times 10^{6}$ & $2.5 \times 10^{2}$ & $9.5 \times 10^{8}$ \\
\hline \hline
\end{tabular}

${ }^{\mathrm{a}}$ Measured as the field level decays below $1 \%$.

grooves in the horizontal and vertical plane as shown in 1(a) not to intersect with the neighbored beam pipe.

Finally, the tools for milling the inner cavity require a minimum diameter of $52 \mathrm{~mm}$ to ensure a tolerance of $\pm 0.1 \mathrm{~mm}$. Hence, the radii $r_{\mathrm{m} 2}, r_{\mathrm{m} 3}$, and $r_{3}$ cannot be smaller than $26 \mathrm{~mm}$.

The rf design has been optimized in several iterations using HFSS and CATIA. The main parameter of the final design are summarized in Table I together with those of the DQWCC, RFDCC, 4RCC, and QMiR. Notice, the different operating frequency of the QMiR requires the individual parameters to be scaled, accordingly, before comparing. The dimensions given in Table I do not account for the wall thickness. A taper angle of $\alpha_{\text {tap }}=30$ deg has been chosen in order to facilitate the $\mathrm{Nb}$ coating process. The overall length of the WOWCC from flange to flange is about $1.4 \mathrm{~m}$. At this length the fundamental mode field exponentially decays to a level at which a flange or any other normal conducting termination does not affect significantly the cavity frequency nor the expected cavity power loss at 4.5 K. The deflecting voltage with respect to the peak surface fields is about $30 \%$ lower than for the RFDCC [7] primarily due to the localized peak of the surface magnetic field as shown in Fig. 3(b). The transverse geometric shunt impedance $(R / Q)_{\perp}$ is lower than for all existing bulk $\mathrm{Nb}$ HL-LHC crab cavity prototypes and allows for a comparably higher loaded quality factor, thus, a larger distance between the fundamental mode coupler and the cavity center. Finally, the sextupolar component is approximately one order of magnitude smaller than provided by the DQWCC, 4RCC, and RFDCC [20]. Note for the QMiR, the multipolar components of the fundamental have not been considered in the design process due to the small beam size in the APS/SPX project [12].

\section{RF POWER LOSS}

Typically, the surface resistance of superconducting cavities shows a nonlinear dependence on the surface magnetic field. Several models have been developed and investigated in order to describe the field dependence such as the breaking of Cooper pairs [23], the thermal feedback [24], or the impurity scattering $[23,25]$. None of them are widely accepted in the literature and in particular for $\mathrm{Nb}$ coated cavities contradicting at least in part experimental results. For this reason, it is most suitable to base the power loss calculation on appropriate measurement data such as those from LHC rf accelerating cavities [26], which likewise employ $\mathrm{Nb} / \mathrm{Cu}$ coating techniques and operate at the same frequency of $400 \mathrm{MHz}$. The measurement of the surface resistance $R_{S}$ is carried out indirectly via the quality factor $Q$ and the geometry factor $G$ according to:

$$
Q=\frac{G}{R_{s}}, \quad \text { with } \quad G=\omega \mu_{0} \frac{\iiint B(\mathbf{r})^{2} d V}{\oiint B(\mathbf{r})^{2} d S} .
$$

The latter one is derived from computational rf simulations. The magnetic field is integrated within the cavity volume or, respectively, over the cavity boundary. Note, we would rather use the magnitude of the magnetic induction $B(\mathbf{r})$ instead of the magnetic field intensity $H(\mathbf{r})$ as it is more common in the SRF community. Both quantities are equivalent since the permeability of free space $\mu_{0}$ applies inside the superconducting rf cavity. Figure 4 shows measurements of a LHC cavity at $4.5 \mathrm{~K}$ and at different peak magnetic surface field which is typically considered as argument. Consequently, the surface resistance $R_{s}\left(B_{p k}\right)$ as it is defined by (3) is an averaged quantity that involves the specific field distribution on the cavity surface. In order to apply the same model on the WOWCC it is necessary, first, to derive the surface resistance localized at the surface element $d S$ as a function of the present field magnitude $B(\mathbf{r})$. Let this quantity be denoted as $R_{s}^{*}(B)$, we have to solve the following inverse problem:

$$
R_{s}\left(B_{p k}\right) \oiint B(\mathbf{r})^{2} d A=\oiint R_{s}^{*}[B(\mathbf{r})] B(\mathbf{r})^{2} d S .
$$




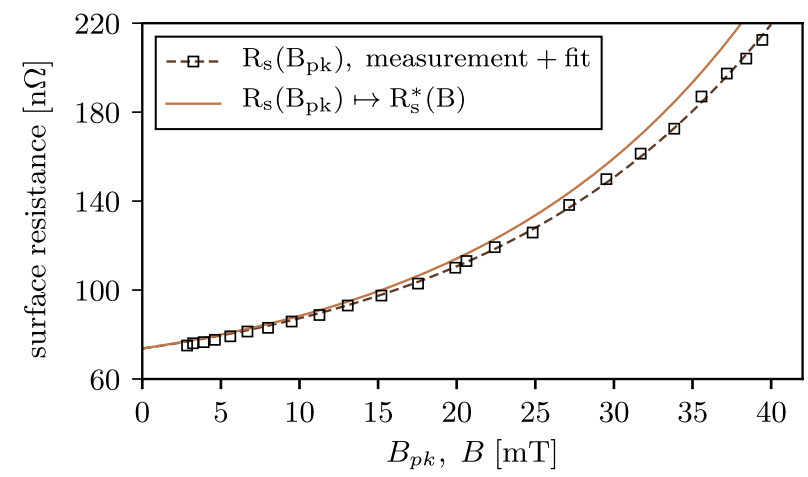

FIG. 4. Surface resistance of a LHC accelerating cavity at $4.5 \mathrm{~K}$ and at $400 \mathrm{MHz}$. In black, the measurement data taken from [26] as a function of the peak magnetic field $B_{p k}$. In dashed brown, an exponential curve fit of the measurements and in light brown, the corresponding localized surface resistance as a function of the present magnetic field $B$.

Once $R_{s}^{*}(B)$ is known, it can be directly used for example in HFSS to evaluate the rf power loss of the WOWCC. It should be mentioned that this approach is still very idealized since it assumes homogeneous surface properties that are exactly the same between the LHC rf accelerating cavity and the WOWCC while in reality the surface quality varies even within one series of coated cavities. Furthermore, the expected peak surface magnetic field is above the available LHC measurement data yielding a further uncertainty by extrapolation. Nevertheless, the mapping $R_{s}\left(B_{p k}\right) \mapsto R_{s}^{*}(B)$ provides some insight into the influence of the surface field distribution on the typical surface resistance measurement. For this purpose three different methods have been applied and are discussed in the following.

Let the surface resistance be an analytic function of the peak surface field $B_{p k}$. The surface resistance can be represented by the Taylor series:

$$
R_{s}\left(B_{p k}\right)=\sum_{n=0}^{\infty} c_{n} B_{p k}^{n}, \quad c_{n} \in \mathbb{R}
$$

Alternatively for the LHC measurement data, it is suitable to apply an exponential fit of the form:

$$
R_{S}\left(B_{p k}\right) \simeq c_{0}+c_{1}\left[\exp \left(c_{2} B_{p k}\right)-1\right],
$$

as shown by the dashed curve in Fig. $4\left(c_{0}=73.7 \mathrm{n} \Omega\right.$, $c_{1}=19 \mathrm{n} \Omega$, and $\left.c_{2}=541 / \mathrm{T}\right)$. Furthermore, let $R_{s}^{*}(B)$ be in the same function space as $R_{s}\left(B_{p k}\right)$ with yet unknown coefficients $c_{n}^{*} \in \mathbb{R}$. Then, from (4) it follows that $c_{0}=c_{0}^{*}$ which physically corresponds to the field independent part of the surface resistance and is given by $R_{\mathrm{BCS}}$ [27] and the residual resistance [28].

To evaluate the remaining coefficients $c_{1}^{*}, c_{2}^{*}, \ldots$, the first approach is based on minimizing the residual from (4) by means of numerical optimization methods such as the Levenberg-Marquardt algorithm. We implemented an optimizer in HFSS as a postprocessing script to evaluate the coefficients $c_{1}^{*}$ and $c_{2}^{*}$ supposing $R_{s}^{*}(B)$ can be well represented by an exponential function of an analogue form of (6).

The second approach is easiest to evaluate for the Taylor series representation (5) and an analog representation of $R_{s}^{*}(B)$ with coefficients $c_{n}^{*}$. Inserting both expressions into (4) and equating coefficients yield:

$$
c_{n}^{*}=c_{n} B_{p k}^{n} \frac{\oiint B^{2}(\mathbf{r}) d A}{\oiint B^{n+2}(\mathbf{r}) d A} .
$$

The third approach requires the surface density function with respect to the magnetic field $B$ similar to $[24,29]$. Equation (4) can be rewritten as:

$R_{s}\left(B_{p k}\right) \int_{0}^{1} u^{2} \sum_{k} \frac{d S_{k}}{d u} d u=\int_{0}^{1} R_{s}^{*}\left(u B_{p k}\right) u^{2} \sum_{k} \frac{d S_{k}}{d u} d u$,

with $u=B / B_{p k}$ and the piecewise analytic defined surface density function $\frac{d S}{d u}=\sum_{k} \frac{d S_{k}}{d u}$. The sum succeeds over local density functions spatially separated by local field extrema. Not all computational simulation tools provide enough information to reconstruct the surface density function for a given field pattern such as shown in Fig. 5. For this reason, we elaborated a generally applicable method which turned out to be more precise than the built-in method available in CST. First, the surface magnetic field together with the coordinate information is exported from the simulation tool with preferably high resolution (e.g., $10^{6}$ points). After, resampling the point cloud (e.g., down to $10^{5}$ points), the surface is reconstructed using the ball pivoting algorithm [30]. Finally, the field information is mapped onto the surface such that both pieces of information are available, the local surface field and the corresponding surface

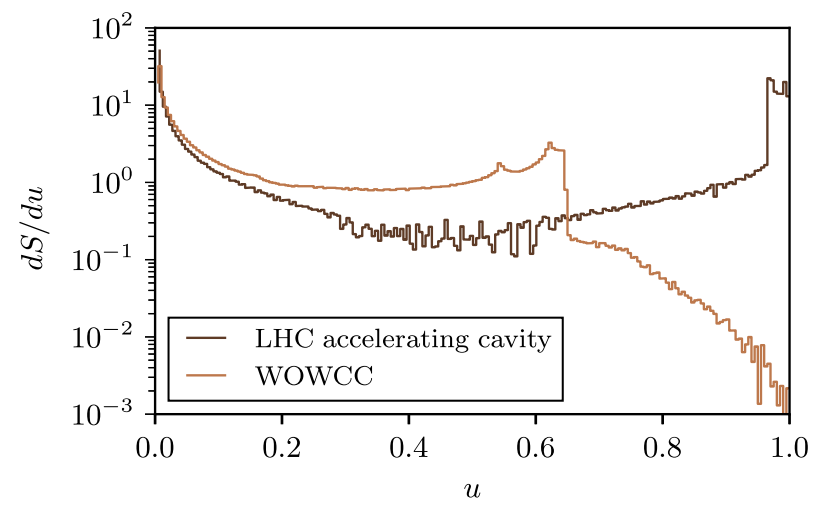

FIG. 5. Surface density function versus surface magnetic field $u=B / B_{p k}$ of the fundamental mode. 
TABLE II. rf power loss of the WOWCC at $V_{\perp}=3 \mathrm{MV}$ using different field dependent surface resistances.

\begin{tabular}{lcc}
\hline \hline$R_{s}^{*}(B)$ & $P_{\text {diss }}$ & $Q_{0}$ \\
\hline $250 \mathrm{n} \Omega$ & $60 \mathrm{~W}$ & $4.3 \times 10^{8}$ \\
LHC model: $\left.R_{s}\left(B_{p k}\right)\right|_{B_{p k}=B}$ & $57 \mathrm{~W}$ & $4.6 \times 10^{8}$ \\
LHC model: $R_{s}\left(B_{p k}\right) \mapsto R_{s}^{*}(B)$ & $65 \mathrm{~W}$ & $4.0 \times 10^{8}$ \\
\hline \hline
\end{tabular}

element area. The remaining steps to evaluate $R_{s}^{*}(B)$ are identical to the second approach, hence inserting the Taylor series into (8). This procedure is interesting insofar as it does not require anymore simulation tools once the surface density function is known.

All three approaches are in good agreement. Interestingly, the resulting local surface resistance $R_{s}^{*}(B)$ is slightly larger than the measured $R_{s}\left(B_{p k}\right)$ as shown in Fig. 4. The same tendency even more pronounced was observed for a HIE-ISOLDE quarter wave resonator [31]. Table II lists the resulting rf power loss of the WOWCC using the local surface resistance obtained from the measurement data of the LHC $\mathrm{rf}$ accelerating cavity in comparison with the two cases already presented in [9]. The resulting intrinsic quality factor of $4 \times 10^{8}$ is slightly lower than the corresponding values of the former study due to the underestimation of the local surface resistance.

\section{BEAM COUPLING IMPEDANCE AND HOMS}

The smooth 30 deg tapers and the large cross section beam pipes provide naturally efficient HOM damping and low beam coupling impedances. Table III lists the detailed results of CST wakefield simulations using an RMS bunch length of $\sigma=80 \mathrm{~mm}$. The corresponding beam coupling impedances are shown in Fig. 6. The effective longitudinal and transverse impedances are calculated according to [32] using the LHC revolution frequency of $11.254 \mathrm{kHz}$. The contribution from the fundamental mode to the transverse kick factor in the deflecting direction $k_{\perp, x}$ is removed to account for parasitic effects, only. Note, the subscript $x$ is related to the deflecting direction while $y$ relates to the non-deflecting direction. The WOWCC approximately provides half the impedance of the DQWCC and a third of the RFDCC, both longitudinally and transversely. Further studies showed that the fundamental mode coupler

TABLE III. Loss factors and effective impedances.

\begin{tabular}{lcccc}
\hline \hline Parameter & Unit & WOWCC & DQWCC & RFDCC \\
\hline$k_{\|}$ & {$[\mathrm{V} / \mathrm{pC}]$} & 0.012 & 0.047 & 0.059 \\
$k_{\perp, x}$ & {$[\mathrm{~V} /(\mathrm{pCm})]$} & 1.242 & 3.051 & 3.357 \\
$k_{\perp, y}$ & {$[\mathrm{~V} /(\mathrm{pCm})]$} & 0.460 & 2.331 & 2.314 \\
$\left(Z_{\|} / n\right)_{\text {eff }}$ & {$[\mathrm{m} \Omega]$} & 0.973 & 1.960 & 2.554 \\
$\left(Z_{\perp, x}\right)_{\text {eff }}$ & {$[\mathrm{k} \Omega]$} & 1.563 & 3.381 & 5.234 \\
$\left(Z_{\perp, y}\right)_{\text {eff }}$ & {$[\mathrm{k} \Omega]$} & 0.438 & 2.177 & 2.253 \\
\hline \hline
\end{tabular}

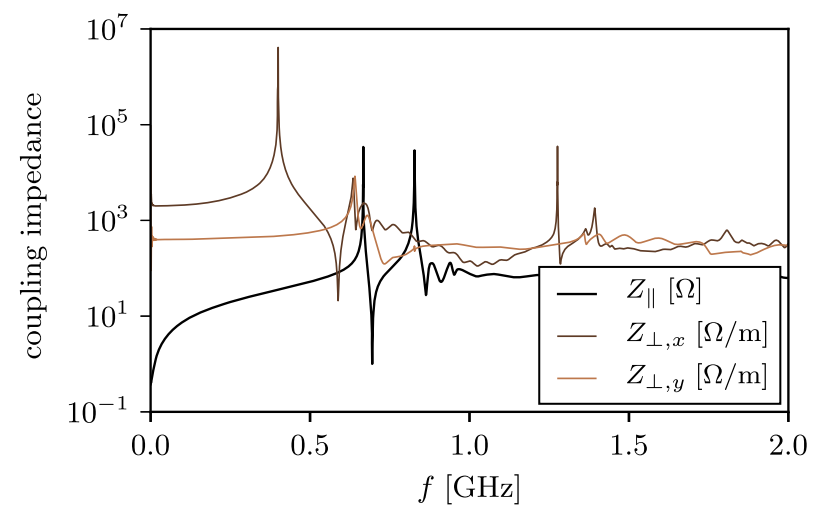

FIG. 6. Longitudinal and transverse beam coupling impedances of the WOWCC.

considered in the next section has only a marginal impact on the impedances. While the longitudinal impedance increases by $2 \%-5 \%$, the transverse impedance decreases by $10 \%-30 \%$ depending on the coupler dimension. A first estimate of the maximum feasible external quality factors $Q_{\text {ext }}$ for HOMs is listed in Table IV. The values are based on the impedance constraints of HL-LHC [3] as well as on the loss and kick factors of the longitudinal and transverse wake potentials, respectively.

Very few modes below $2.5 \mathrm{GHz}$ have been identified by eigenmode simulations using CST and HFSS as expected from the beam coupling impedances. The detailed results are given in Table V. The first two higher-order dipole modes are of less concern since their frequency around $640 \mathrm{MHz}$ is above the cutoff frequency of the corresponding waveguide mode, they couple to. Such propagating

TABLE IV. Max. required $Q_{\text {ext }}$ based on HL-LHC data [3].

\begin{tabular}{lcc}
\hline \hline Mode type & Peak real impedance & $Q_{\text {ext }}$ threshold \\
\hline monopole & $2.4 \mathrm{M} \Omega$ & $2.02 \times 10^{5}$ \\
dipole & $1.5 \mathrm{M} \Omega / \mathrm{m}$ & $1.25 \times 10^{3}$ \\
\hline \hline
\end{tabular}

TABLE V. HOMs of the WOWCC.

\begin{tabular}{|c|c|c|c|c|c|}
\hline $\begin{array}{l}\text { Cavity } \\
\text { mode }^{\text {a }}\end{array}$ & $\begin{array}{c}f \\
{[\mathrm{MHz}]}\end{array}$ & $\begin{array}{l}R / Q \\
{[\Omega]^{\mathrm{b}}}\end{array}$ & $\begin{array}{l}\text { Beam pipe } \\
\text { mode }^{\mathrm{a}}\end{array}$ & $\begin{array}{l}\text { Cut-off } \\
{[\mathrm{MHz}]}\end{array}$ & $Q_{\mathrm{ext}}^{{ }^{\mathrm{c}}}$ \\
\hline $\mathrm{TE}_{111}$ & 400.0 & 171.85 & $\mathrm{TE}_{11}$ & 624.9 & $1.0 \times 10^{6}$ \\
\hline $\mathrm{TE}_{112}$ & 638.3 & 7.85 & $\mathrm{TE}_{11}$ & 624.9 & $<35$ \\
\hline $\mathrm{TE}_{111}$ & 643.8 & 0.04 & $\mathrm{TE}_{11}$ & 624.9 & $<40$ \\
\hline $\mathrm{TE}_{012}$ & 667.0 & 6.95 & $\mathrm{TM}_{01}$ & 847.6 & $4.9 \times 10^{4}$ \\
\hline $\mathrm{TM}_{011}$ & 827.2 & 12.55 & $\mathrm{TM}_{01}$ & 847.6 & $1.4 \times 10^{4}$ \\
\hline $\mathrm{TE}_{111}$ & 1276 & 0.15 & $\mathrm{TE}_{21}$ & 1180 & $1.1 \times 10^{3}$ \\
\hline
\end{tabular}

${ }^{\mathrm{a}}$ Mode indices in analogy to the pillbox or circular wave guide.

${ }^{\mathrm{b}}$ For monopole modes, the longitudinal $(R / Q)$ while for dipole modes, the transverse $(R / Q)_{\perp}$, both in circuit definition.

${ }^{\mathrm{c}}$ Damping via the wide opened apertures, the fundamental mode coupler, and the HOM coupler as shown in Fig. 8. 
HOMs are considered to be damped outside the cryomodule, for example, via ferrites. There are only three HOMs which require additional damping strategies. Their electric and magnetic field distributions are shown in Figs. 7(a)-7(f). First attempts to mitigate their impact on the beam dynamic envisaged simple probe antennas without notch filter and away from the cavity center. However, this approach is not sufficient with respect to the dipole mode at $1276 \mathrm{MHz}$ which partially provides a field distribution similar to that of the fundamental mode. By comparing all three HOMs, the most appropriate way to couple to all of them is given by magnetic coupling at the

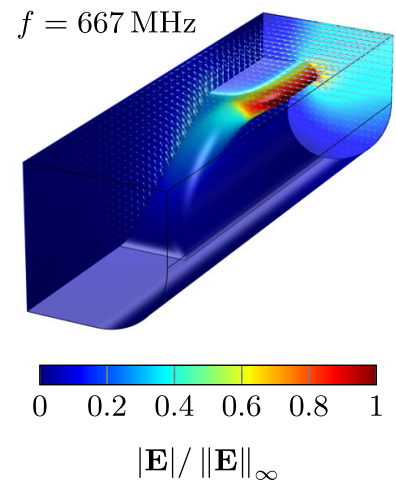

(a)

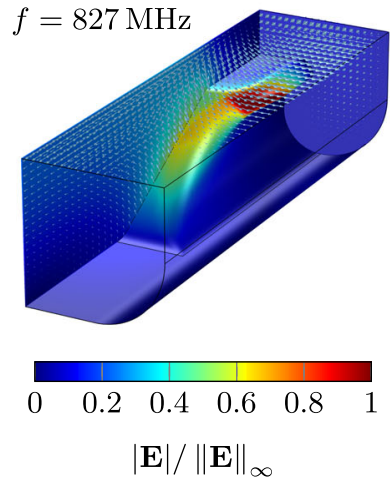

(c)

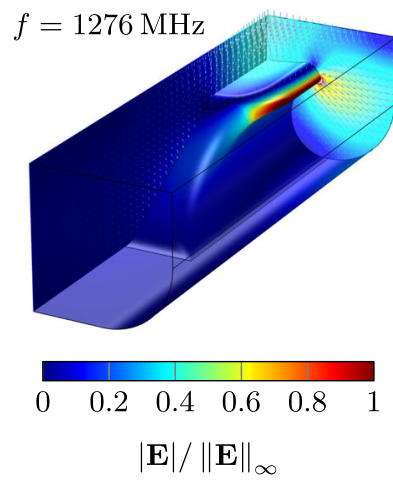

(e)

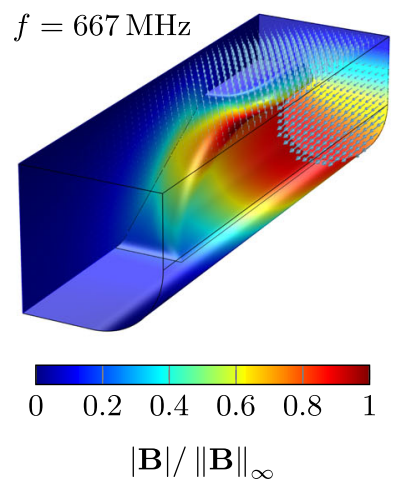

(b)

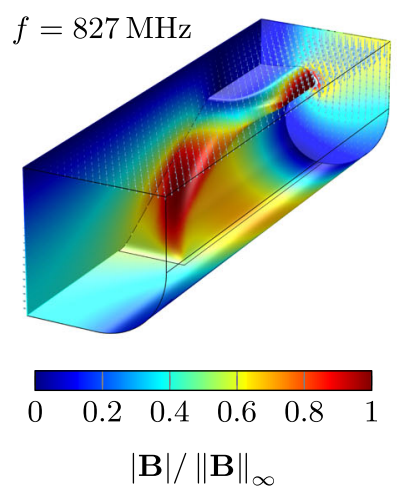

(d)

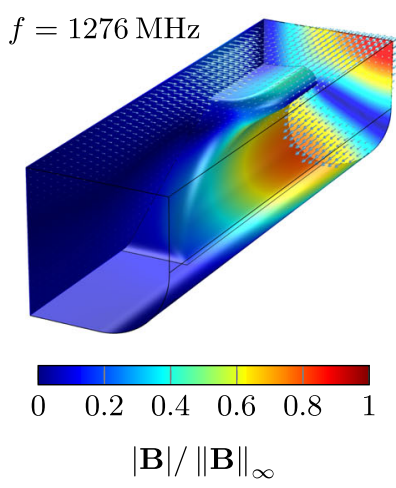

(f)
FIG. 7. Normalized electric and magnetic field distributions of the trapped HOMs. cavity center. The hook antenna should be somewhat transversely (vertically) displaced from the center in order to allow coupling to the monopole mode at $667 \mathrm{MHz}$ [Fig. 7(b)]. At this position, since the magnetic field of the second monopole HOM at $827 \mathrm{MHz}$ is oriented exactly perpendicular to those of the other HOMs, the hook antenna must be mounted skewed as shown in Fig. 8 . Furthermore, the HOM coupler requires a notch filter not to couple to the fundamental mode.

The slightly modified version of the LHC DQWCC HOM coupler [33] shown in Figs. 8 and 9 has been adjusted, in particular, to the second and third HOM at $827 \mathrm{MHz}$ and $1276 \mathrm{MHz}$, respectively. The resulting external quality factors $Q_{\text {ext }}$ listed in Table V fairly satisfy the requirements. They also include the damping through the beam pipe and the fundamental mode coupler, however, both have only marginal influence on the trapped HOMs. It is worthwhile to note that in contrast to the DQWCC and the RFDCC, only a single coupler suffices for the HOM

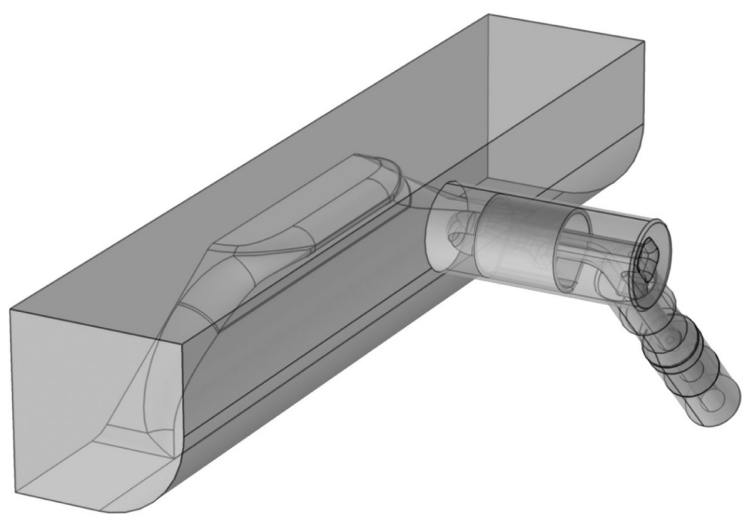

FIG. 8. A quarter of the WOWCC with an HOM coupler similar to those used for the DQWCC [33]. The coupler is located at the cavity center vertically displaced by $40 \mathrm{~mm}$ and rotated by 45 deg.

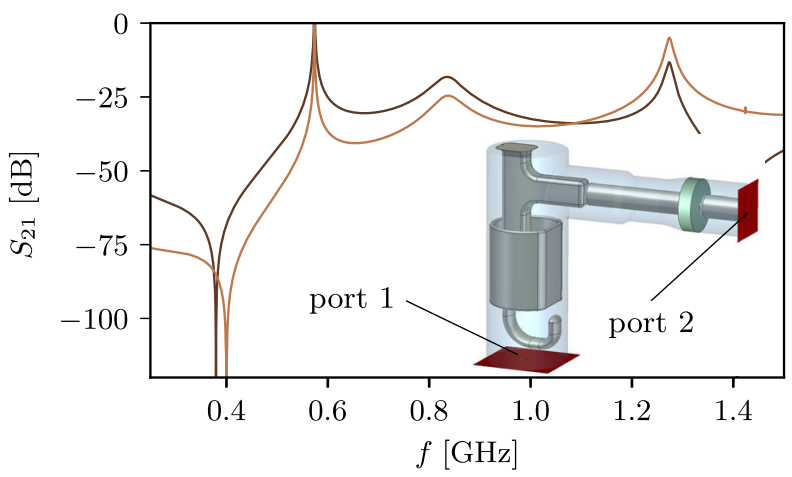

FIG. 9. Scattering parameters of the adjusted LHC DQWCC HOM coupler. In dark brown, transmission between the $\mathrm{TM}_{01}$ mode at port 1 and the TEM mode at port 2. In light brown, transmission between a $\mathrm{TE}_{11}$ mode at port 1 and the TEM mode at port 2 . 
power extraction. A detailed HOM coupler design as well as the assessment of damping mechanisms outside the cryomodule related to propagating HOMs are foreseen for a future redesign.

\section{FUNDAMENTAL MODE COUPLER}

The fundamental mode is coupled via the electric field in the deflecting direction close to the taper as shown in Fig. 10. A probe antenna can be used instead of a hook antenna which is likewise favored from the thermal point of view. The optimal coupling is derived from the input power required to compensate beam loading if the beam is off-centered. Let $\Delta x$ be the displacement of the beam from the center in the deflecting direction. The required input power calculates as [34]:

$$
P_{\mathrm{g}}=\frac{1}{2}(R / Q)_{\perp} Q_{\mathrm{ext}}\left|I_{\mathrm{g}}\right|^{2},
$$

where

$I_{\mathrm{g}}=\frac{V_{\perp}}{2(R / Q)_{\perp} Q_{\mathrm{ext}}}+\frac{\omega \Delta x}{c_{0}} I_{\mathrm{b}, \mathrm{DC}} F_{\mathrm{b}}-j \frac{V_{\perp} \Delta \omega}{\omega(R / Q)_{\perp}}$,

with the transverse geometric shunt impedance in linac definition $(R / Q)_{\perp}$, the adequate DC beam current $I_{\mathrm{b}, \mathrm{DC}}$ weighted by the bunch form factor $F_{\mathrm{b}}$, and the angular frequency deviation $\Delta \omega$ between cavity resonance and the excitation wave from the coupler. The latter one is assumed to be zero in the calculations shown in Fig. 11, while the beam displacement is expected to be within $\Delta x= \pm 2 \mathrm{~mm}$. The minimum appears at around $Q_{\mathrm{ext}}=1 \times 10^{6}$ which means a slightly lower coupling than considered for the three bulk $\mathrm{Nb}$ crab cavities due to their higher $(R / Q)_{\perp}$ values for the fundamental mode [3]. This is interesting in that the fundamental mode coupler can be located farther away from the cavity center.

Three different probe antennas denoted as $\mathrm{C} 1, \mathrm{C} 2$, and C3 are compared in Table VI, each of them adjusted to a coupling of $Q_{\mathrm{ext}}=1 \times 10^{6}$. The first two agree in their dimensions with those used for the accelerating cavities in LHC or SPL [35], respectively. The latter is originated from the fundamental mode coupler of the DQWCC, by removing the hook (Fig. 10). The power dissipated on the coupler

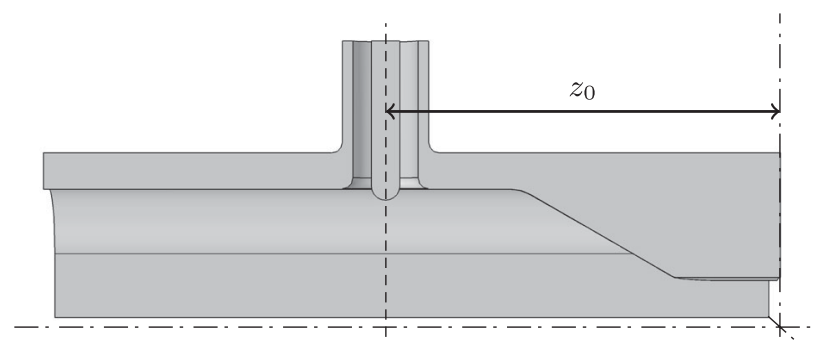

FIG. 10. Fundamental mode coupler close to the 30 deg taper.

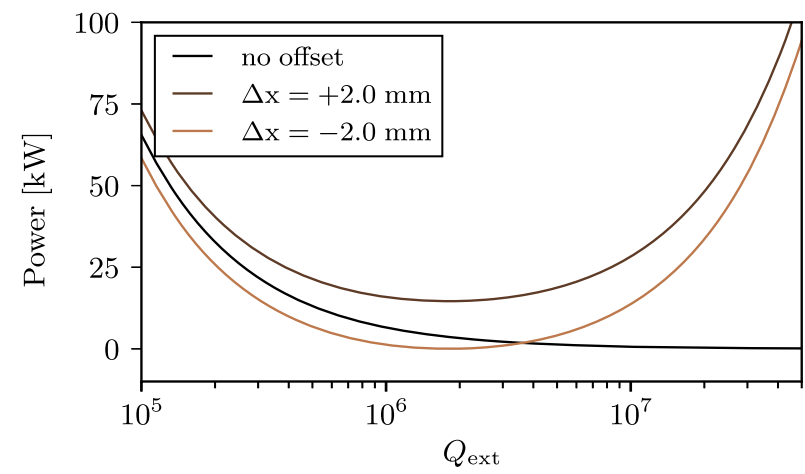

FIG. 11. Input rf power as a function of the external quality factor of the fundamental mode. The beam is offset in the deflecting direction by maximum $\pm 2 \mathrm{~mm}$. The bunch form factor at $400 \mathrm{MHz}$ is approximately 1 [3]. An LHC DC beam current of $0.58 \mathrm{~A}$ is assumed.

surface is related to the surface resistance of $\mathrm{Cu}$ at $400 \mathrm{MHz}$ and at room temperature. The peak surface fields as well as dissipated powers throughout all designs are feasible. It is noteworthy that these values are about one order of magnitude smaller than those of the DQWCC fundamental mode coupler [36]. In agreement with the power requirements of the DQWCC and RFDCC, the smallest coupler (C3) has been selected which is also favored from the mechanical point of view.

The cavities' dipolar and sextupolar components are not influenced by the fundamental mode coupler as can be seen in Table VII. A skew quadrupolar component $a_{2}$ is introduced, however its value is marginal.

TABLE VI. Properties of fundamental mode couplers.

\begin{tabular}{lcccc}
\hline \hline Parameter & Unit & $\mathrm{C} 1$ & $\mathrm{C} 2$ & $\mathrm{C} 3$ \\
\hline inner diameter & {$[\mathrm{mm}]$} & 41.0 & 43.0 & 27.0 \\
outer diameter & {$[\mathrm{mm}]$} & 145.0 & 100.0 & 62.0 \\
tip rounding & {$[\mathrm{mm}]$} & 20.5 & 5.0 & 13.5 \\
insertion & {$[\mathrm{mm}]$} & 0.0 & 0.0 & 8.0 \\
distance from center $z_{0}$ & {$[\mathrm{~mm}]$} & 467 & 429 & 375 \\
$E_{p k}$ at $V_{\perp, 0}$ & {$[\mathrm{MV} / \mathrm{m}]$} & 0.545 & 0.702 & 1.937 \\
$B_{p k}$ at $V_{\perp, 0}$ & {$[\mathrm{mT}]$} & 1.12 & 1.73 & 3.95 \\
$P_{\text {diss }}\left(R_{S}=5.2 \mathrm{~m} \Omega\right)$ & {$[\mathrm{W}]$} & 3.45 & 8.21 & 12.92 \\
\hline \hline
\end{tabular}

TABLE VII. Multipolar moments with and without fundamental coupler at a total deflecting voltage of $10 \mathrm{MV}$.

\begin{tabular}{lcccc}
\hline \hline & Unit & No coupler & $\mathrm{C} 1$ & $\mathrm{C} 3$ \\
\hline$\Re\left\{b_{1}\right\}$ & {$[\mathrm{Tm}]$} & $3.34 \times 10^{-2}$ & $3.34 \times 10^{-2}$ & $3.34 \times 10^{-2}$ \\
$\Im\left\{b_{1}\right\}$ & {$[\mathrm{Tm}]$} & $\ldots$ & $2.86 \times 10^{-5}$ & $5.92 \times 10^{-6}$ \\
$\Im\left\{a_{2}\right\}$ & {$[\mathrm{Tm} / \mathrm{m}]$} & $\ldots$ & $3.78 \times 10^{-4}$ & $8.61 \times 10^{-5}$ \\
$\Re\left\{b_{3}\right\}$ & {$\left[\mathrm{Tm} / \mathrm{m}^{2}\right]$} & $9.01 \times 10^{-2}$ & $8.81 \times 10^{-2}$ & $8.88 \times 10^{-2}$ \\
$\Re\left\{b_{5}\right\}$ & {$\left[\mathrm{Tm} / \mathrm{m}^{4}\right]$} & $1.41 \times 10^{3}$ & $1.41 \times 10^{3}$ & $1.41 \times 10^{3}$ \\
\hline \hline
\end{tabular}




\section{MULTIPACTING}

Multipacting, the phenomenon of an avalanchelike growth of free electrons, first observed by Henneberg [37], occurs frequently during the rf conditioning of cavities and couplers. It requires free electrons to be resonantly driven by an rf field such that the cavity wall or coupler surface is repetitively impacted at nearly the same locations. If the material provides a secondary electron yield (SEY) larger than one at the corresponding impact energy of the present primary electrons, the process can lead to a resonant growth of free electrons. As a result, the temperature rapidly rises at the affected locations and eventually leads to an excessive rf loss. Some SEY curves of $\mathrm{Nb}$ are shown in Fig. 12. Since multipacting may considerably extend the processing time of an rf device (soft barriers) or even limit its performance (hard barrier), it should be assessed in the design phase. A general study of multipacting in the iris region of rf deflecting cavities using an analytic model is given in [38]. The results found in there correspond to the first barrier discussed in this section.

The multipacting barriers of the bare cavity have been characterized using both, CST and ACE3P [39]. CST offers two options by combining the rf eigenmode solver either with the particle tracking solver or with the particle-in-cell solver (PIC solver). The electron growth rate is analyzed explicitly by the time averaged ratio of secondary emitted electrons from the cavity surface to the primary electrons:

$$
\text { growth rate }=\left\langle\frac{I_{\mathrm{SEE}}}{I}\right\rangle,
$$

and implicitly by an exponential fit of the total electron number over the time according to:

$$
\frac{I\left(t_{2}\right)}{I\left(t_{1}\right)}=\text { growth rate }{ }^{(2 f \Delta t / n)},
$$

where $n$ corresponds to the order of multipacting. Many publications such as $[38,40,41]$ throughout refer to the first

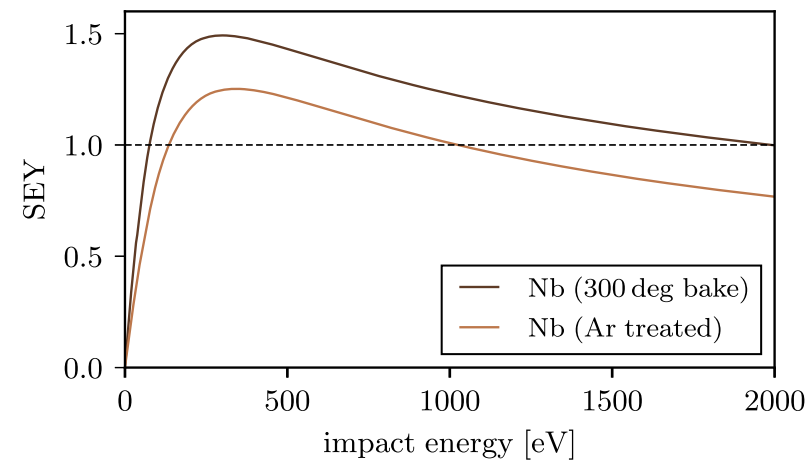

FIG. 12. Secondary electron yield (SEY) of $\mathrm{Nb}$ after $300^{\circ} \mathrm{C}$ baking and after a surface treatment with argon [15]. option using the particle tracking solver though it requires additional parameter sweeps through rf phases. In contrast, the PIC solver used within this study emits an entire particle bunch from the surface covering a range of different $\mathrm{rf}$ phases. Note, some useful built-in post processing scripts to analyze the growth rate are only available for the particle tracking solver but can be adapted to the needs of the PIC solver. ACE3P offers the combination of the rf eigenmode solver OMEGA3P and the tracking sover TRACK3P. Here, the impact energy of resonant particles that repetitively hit the surface at nearly the same location is compared to the energy range in which the material dependent secondary electron yield is larger than one. For $\mathrm{Nb}$, this range lies approximately between $80 \mathrm{eV}$ to $2000 \mathrm{eV}$ but the actual SEY curve strongly depends on the surface preparation. The SEY of $\mathrm{Nb}$ after $300^{\circ} \mathrm{C}$ baking (Fig. 12) taken from the material library of CST shall serve as a reference in the following. To keep the required computing resources for both tracking codes feasible, the cavity wall was partitioned into six slices along the longitudinal direction to localize the initial particle emission as illustrated in Fig. 13. Moreover, only half the cavity is considered. The particles were tracked over $70 \mathrm{rf}$ cycles in ACE3P and over 10 to 100 rf cycles in CST depending on the growth rate.

Three multipacting barriers between $0.2 \mathrm{MV}$ to $2.0 \mathrm{MV}$ deflecting voltage have been identified using ACE3P (Figs. 14 and 15). According to the Gaponov-Miller force [42], the resonant particle trajectories appear at distinct electric field minima which becomes obvious by observing the rescaled electric field intensity on the upper left in Fig. 14(a). The first barrier at a deflecting voltage between $V_{\perp}=0.2-0.7 \mathrm{MV}$ is driven by resonant particles between the two ridges close to the cavity center. Likewise close to the cavity center but at the top and bottom appears multipacting between $V_{\perp}=1.0-$ 1.5 MV causing the second barrier whereas an electron growth further away from the ridges provides a third barrier at even higher field level. The separate consideration of several initial electron emitters allows for precise qualitative and quantitative characterizations of the multipacting barriers, in particular, the change of the energy range that favors

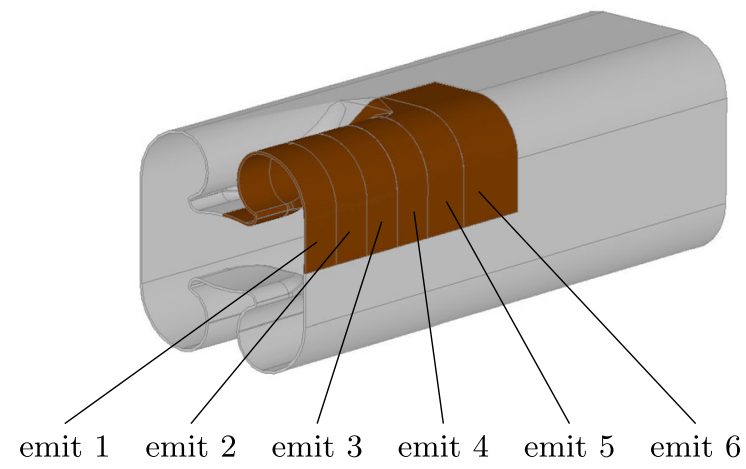

FIG. 13. The partition of the cavity surface to provide local initial field emission. 


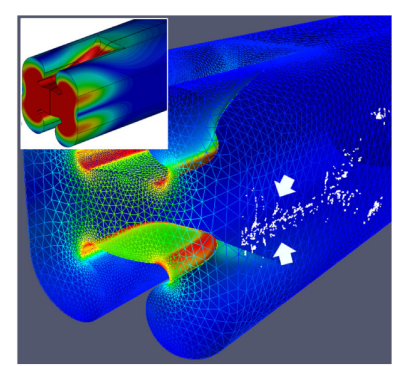

(a)

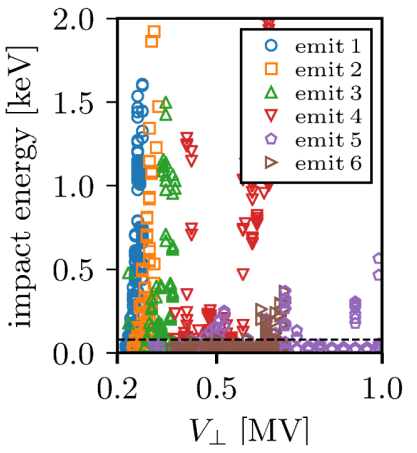

(b)
FIG. 14. First multipacting barrier at $V_{\perp}=0.2-0.7 \mathrm{MV}$. (a) Electric field at the cavity surface with the resonant particles trajectories after $70 \mathrm{rf}$ cycles (white). (b) Corresponding impact energies for different initial emitter locations. The simulations are carried out using ACE3P.

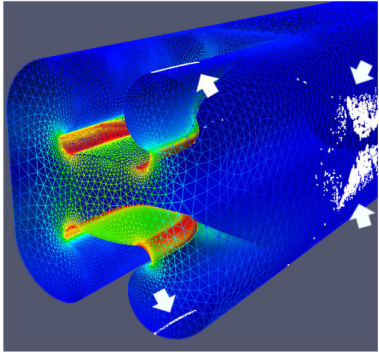

(a)

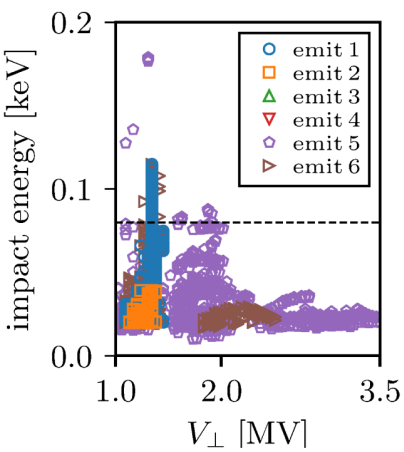

(b)
FIG. 15. Second and third barrier at $V_{\perp}=1-2 \mathrm{MV}$. (a) Electric field at the cavity surface with the resonant particles trajectories after $70 \mathrm{rf}$ cycles (white). (b) Corresponding impact energies for different initial emitter locations. The simulations are carried out using ACE3P.

multipacting the farther the resonant particles are away from the cavity center.

Figures 16(a) and 16(b) show the corresponding results of CST simulations using the SEY curve of $\mathrm{Nb}$ after $300^{\circ} \mathrm{C}$ baking and after a surface treatment with argon, respectively. The same constellation of localized initial field emitters is assumed as before (Fig. 13). In contrast to ACE3P, the second and third barriers are significantly larger whereas the first one is slightly smaller. Furthermore, the transition from the second to the third barrier is rather continuous and not volatile as it might deduced from Fig. 15(a). Thus, the electron growth that appears at lower energies at the top and bottom around the cavity center continuously "moves" with higher impact energies toward the sides farther away from the center. Moreover, the first multipacting barrier completely disappears for the lower SEY curve while the others become significantly weaker which indicates soft multipacting barriers.

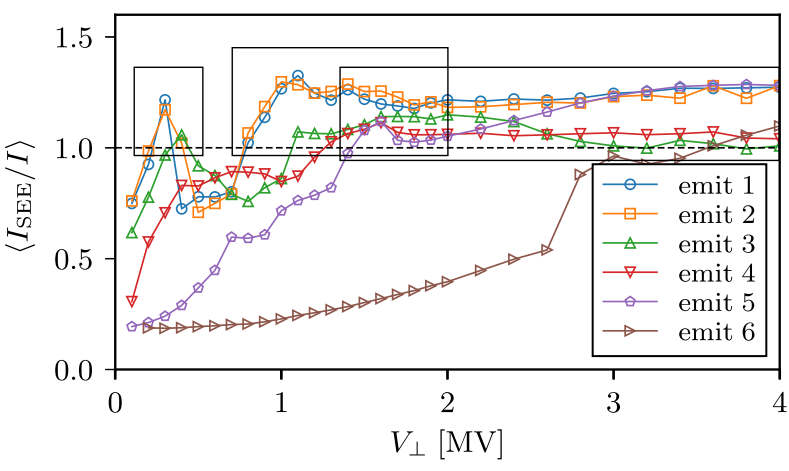

(a)

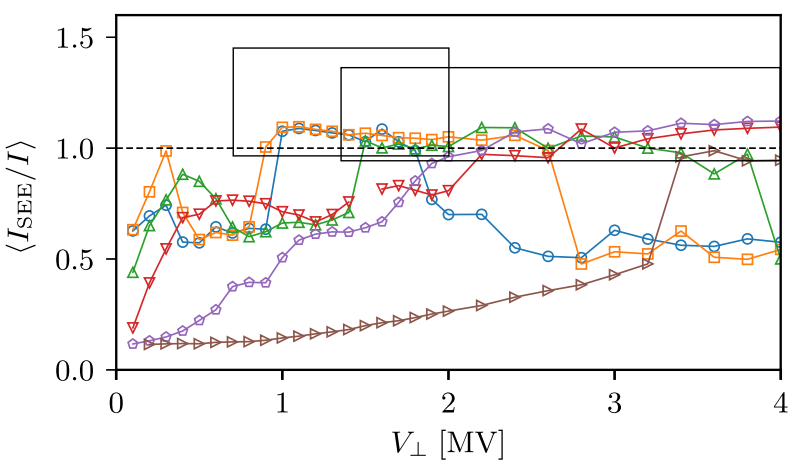

(b)

FIG. 16. Electron growth rate versus impact energy for different emitter locations using the secondary electron yield of $\mathrm{Nb}$, (a) after $300{ }^{\circ} \mathrm{C}$ baking, and (b) after a surface treatment with argon. The three multipacting barriers are highlighted. The simulations are carried out using CST.

The investigations of the RFDCC using ACE3P provide very similar results [43]. The first cold test of the RFDCC prototype revealed only the narrower barrier at the lowest field level which could successfully passed.

\section{RF SENSITIVITY TO PRESSURE}

Depending on the wall thickness and shape, the frequency of the cavity can be highly sensitive to pressure fluctuations in the helium bath. In order to mitigate this aspect, the exterior cavity shape should be defined such that the deformation due to outer pressure variations affects the fundamental mode frequency as low as possible. It is worth noting that the dynamic contribution is considered quasistatic. Figure 17(a) shows a quarter of the transverse cross section at the center including the external shape. For fabrication reasons, the external geometry follows a cylindrical profile with an exterior diameter given by a minimum wall thickness of $8 \mathrm{~mm}$. In order to accommodate neighboring beam pipes in both horizontal and vertical crabbing, grooves with radius $r_{\mathrm{g}}$ are machined out from this cylindrical profile. Figure 17(b) illustrates the principle of optimizing the external shape through $r_{\mathrm{g}}$ such that the impact of pressure fluctuations in the helium bath on the fundamental mode frequency vanishes. For the sake of 


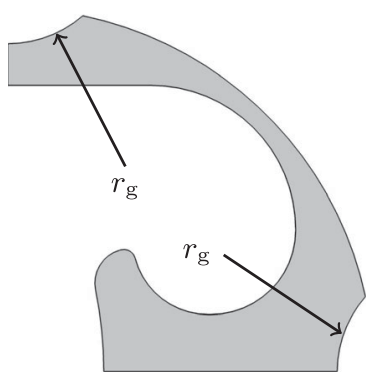

(a)

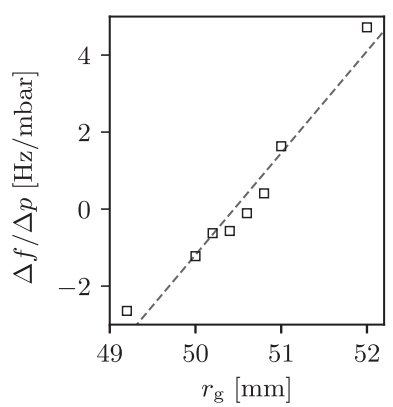

(b)
FIG. 17. (a) Quarter of the transverse cavity cross section with the groove radius $r_{\mathrm{g}}$. (b) The frequency versus pressure sensitivity as a function of the groove radius calculated in CST. A quasistatic pressure difference of 1 bar is applied at the outer cavity wall. Gravity is not considered.

simplicity, the vertical and horizontal groove radii were chosen to be the same.

The exterior shape of the WOWCC has been optimized, first, by means of the 2D transverse cross section at the cavity center [9]. Consequently, the taper and any small cavity features on the exterior surface were not taken into account. Note that for fabrication reasons, the prototype is split into three sections that are welded together. At the interfaces, the wall thickness is reduced to $8 \mathrm{~mm}$. To address the taper at the interior as well as the discontinuities at the exterior cavity surface, the frequency versus pressure sensitivity calculations are extended towards fully coupled 3D simulations in a second step. As the goal of the first prototype is not to have lowest pressure sensitivity but rather to assess manufacturability, coating, and rf performance, the design was frozen even if only suboptimal. The focus in the following lies on the computational tools and methods used. The combination of $\mathrm{rf}$ and mechanical performance is a nontrivial and interesting multiphysics case. These tools will be experimentally validated by rf tests and fully employed for a later prototype.

The frequency versus pressure sensitivity is investigated using four different options to combine an rf eigenmode solver with a structural mechanics solver: (i) CST, (ii) HFSS within the ANSYS workbench [44], (iii) COMSOL, and (iv) APDL [45]. The details of this study are summarized in Fig. 18(a) and 18(b). The scaled deformation depicted in Fig. 19 reveals a maximum displacement at the welds between the three cavity parts. The number of mesh elements in Fig. 18 comprises both, the interior vacuum for the rf simulations and the cavity wall together with the flanges for the structural mechanics simulations. The latter one accounts roughly for $30 \%$ of the total mesh elements. The best agreement of the frequency versus pressure sensitivity is found between COMSOL and APDL despite of the larger absolute frequency errors. APDL offers two macros in order to apply further analyses on a deformed

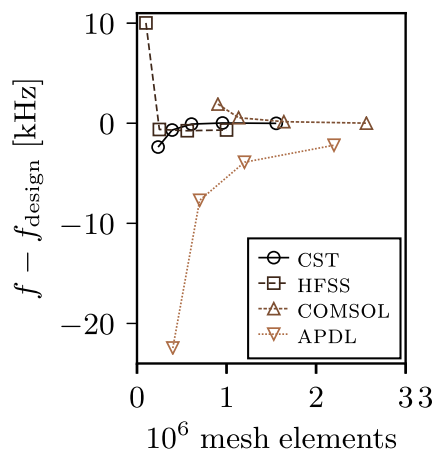

(a)

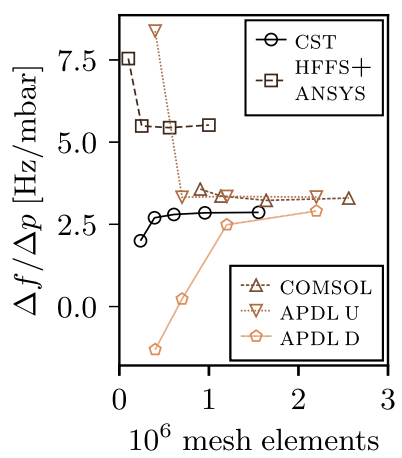

(b)
FIG. 18. Convergence study based on mesh refinement. (a) The frequency error of $\mathrm{rf}$ eigenmode simulation by means of the undeformed geometry. (b) The frequency versus pressure sensitivity derived from a quasistatic pressure difference of 1 bar at the outer cavity wall where both flanges are fixed and gravity is not present. The two methods to apply an rf simulation for a deformed geometry in APDL are denoted as UPGEOM (U) and DVMORPH (D).

geometry: DVMORPH which remeshes the deformed geometry such as used in [46], and UPGEOM which transfers the nodal displacements to the mesh elements directly. The latter one is fairly converged using a mesh of $10^{6}$ elements while the first method requires approximately three times this number yielding a significantly higher memory usage ( $200 \mathrm{~GB})$ and longer solver time. In contrast, the framework provided by HFSS within the ANSYS workbench turns out to be very problematic for complex geometries. The frequency sensitivity to pressure fluctuations is twice as high as predicted by the others. Further mesh refinement above $10^{6}$ elements, in all cases lead to corruptions either in

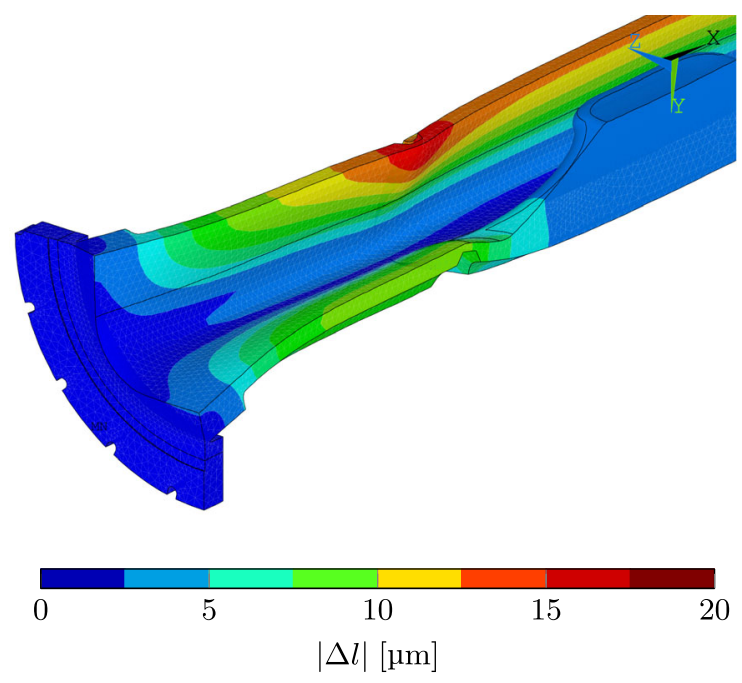

FIG. 19. Deformation $\Delta l$ due to a quasistatic pressure difference at the cavity walls of 1 bar as simulated in APDL. Both flanges are fixed in position and symmetry is applied in the transverse directions. Gravity is not considered. 
TABLE VIII. Maximum deformation for the set-up in Fig. 19.

\begin{tabular}{lccc}
\hline \hline CST & ANSYS+HFSS & COMSOL & APDL \\
\hline $18.39 \mu \mathrm{m}$ & $17.51 \mu \mathrm{m}$ & $17.52 \mu \mathrm{m}$ & $17.46 \mu \mathrm{m}$ \\
\hline \hline
\end{tabular}

TABLE IX. Material properties of $\mathrm{Cu}$.

\begin{tabular}{lccccc}
\hline \hline Property & Unit & $293 \mathrm{~K}$ & $77 \mathrm{~K}$ & $4.2 \mathrm{~K}$ & Ref. \\
\hline $\mathrm{T}$ contraction $\left(\frac{L_{293}-L(T)}{L_{293}}\right)$ & {$\left[10^{-3}\right]$} & 0 & 3.0409 & 3.2567 & {$[47]$} \\
Young's modulus & {$[\mathrm{GPa}]$} & 115.0 & 123.4 & 131.3 & {$[48]$} \\
Poisson ratio & $\cdots$ & 0.34 & 0.34 & 0.34 & \\
\hline \hline
\end{tabular}

the mesh deformation or in the rf eigenmode simulation of the deformed mesh. It is worth noting that similar issues in using HFSS together with ANSYS have been experienced for the LHC rf accelerating cavity. Turning the attention to CST, though the results quickly converge, a residual error of $5 \%-10 \%$ persists due to inaccurate deformation results as confirmed by the CST support. A quantitative comparison of the structural mechanics solvers by means of the maximum deformation at 1 bar quasistatic pressure fluctuation is given in Table VIII.

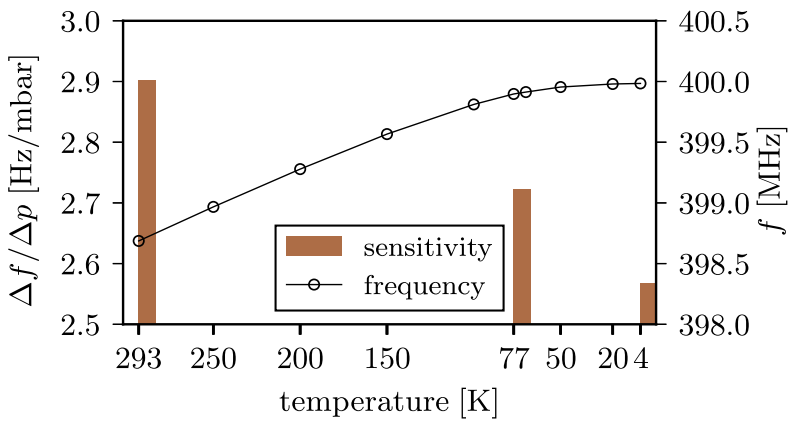

FIG. 20. In brown, the frequency versus pressure sensitivity at different temperatures as simulated in APDL. In black, the corresponding fundamental mode frequency without quasistatic pressure. To mimic the setup of a vertical cold test in a cryostat, the cavity is fixed only at the top flange such that gravity is applied in longitudinal direction.

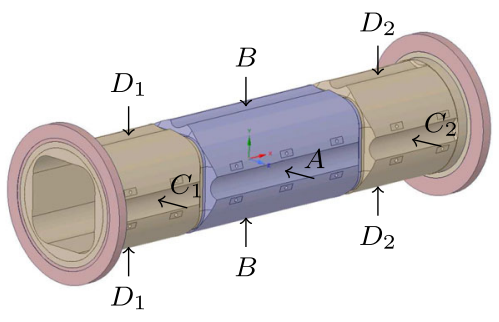

(a)

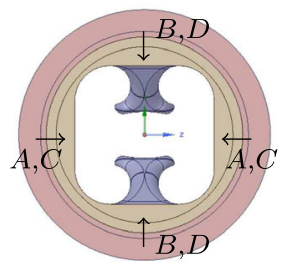

(b)
FIG. 21. Considered tuner positions in (a) isometric view and (b) longitudinal view.
TABLE X. Frequency sensitivity to tuning.

\begin{tabular}{lcccc}
\hline \hline Unit & $A$ & $B$ & $C$ & $D$ \\
\hline$[\mathrm{MHz} / \mathrm{mm}]$ & 1.51 & 2.16 & -0.03 & 0.67 \\
{$[\mathrm{~Hz} / \mathrm{N}]$} & 78.6 & 46.9 & -0.63 & 14.7 \\
\hline \hline
\end{tabular}

Based on the above described analyses, the frequency versus pressure sensitivity of the prototype design was further investigated at different temperatures and in the presence of gravity using APDL, only, though COMSOL offers an equivalent option. According to the material properties listed in Table IX, the cavity becomes slightly less elastic while cooling down. As shown in Fig. 20, the sensitivity reduces from $2.9 \mathrm{~Hz} / \mathrm{mbar}$ at room temperature to $2.58 \mathrm{~Hz} / \mathrm{mbar}$ at $4.2 \mathrm{~K}$ close to the operating temperature. Gravity acts along the cavity as it would be installed in the vertical cold test. The flanges are considered to be of stainless steel with a Young's modulus of $200 \mathrm{GPa}$ and a Poisson ratio of 0.3 independent of the temperature.

The conceptual feasibility of a tuner mechanism is also investigated. Four positions are considered, Fig. 21: (A) one tuner in the plane perpendicular to the symmetry plane of the mushroom-shaped ridges at the very center of the cavity with two opposite contact points, (B) one tuner parallel to the plane of the ridges acting directly on them with two opposite contact points, (C) two perpendicular tuners in the plane perpendicular to the ridges with four contact points, $450 \mathrm{~mm}$ from the center, and (D) two perpendicular tuners in the plane of the mushroom with four contact points, $450 \mathrm{~mm}$ from the center. Table X compares the sensitivity of the cavity for each of the four concepts of tuner configuration and shows that the available choice of sensitivity is rather large.

\section{CONCLUSION}

A novel crab cavity (wide-open-waveguide, WOWCC) has been conceived within the High Luminosity upgrade of the LHC and is now part of the FCC study. Various design aspects of the cavity have been investigated. The optimized interior cavity shape allows for a deflecting voltage of $3 \mathrm{MV}$ at $400 \mathrm{MHz}$ and at peak surface fields of $45 \mathrm{MV} / \mathrm{m}$ and $78 \mathrm{mT}$. The design of the WOWCC is adapted to the needs of the coating process which, in particular, determines the 30 deg taper and the open waveguides.

The field dependence of the surface resistance taking into account the rf loss evaluation provides an interesting insight on the influence of the cavity shape on the measured surface resistance, but does not yield significant changes for the expected intrinsic quality factor. The discussed approaches to mitigate HOM effects are only related to the trapped HOMs. A detailed study with respect to HOM dampers that extract the power of propagating modes outside the cryomodule is still needed. Current activities include a redesign of the WOWCC to maximize $\mathrm{rf}$ 
performance and improve accessibility for coating. Correspondingly, the HOM studies will be reassessed and continued by means of the redesign.

Multipacting barriers have been investigated using CST and $\mathrm{ACE} 3 \mathrm{P}$ and are very likely to appear in the cold test similar to the behavior of the RFDCC.

Finally, a consistent comparison of approaches for estimating the frequency sensitivity to pressure has been performed and shows that ANSYS-APDL and COMSOL are suitable options for this kind of multiphysics analysis. The procedure described and refined for this prototype will be used for the future design iterations.

The proof-of-principle cavity whose design was presented in this article will explore new limits for coated superconducting cavities at $400 \mathrm{MHz}$. A first prototype is being fabricated and tested at CERN and will help direct future $\mathrm{Nb} / \mathrm{Cu}$ related $\mathrm{R} \& \mathrm{D}$ projects.

\section{ACKNOWLEDGMENTS}

The authors would like to thank James Alexander Mitchell for providing a parametric model of the LHC DQWCC HOM coupler. We would also like to acknowledge the fruitful discussions with Akira Miyazaki.

[1] R. B. Palmer, Energy scaling, crab crossing and the pair problem, SLAC Technical Report No. SLAC-PUB-4707, 1988.

[2] K. Hosoyama, K. Hara, A. Kabe, Y. Kojima et al., in Proceedings of Asian Particle Accelerator Conference, APAC'98 (KEK Proceedings 98-10, Tsukuba, Japan, 1998), pp. 828-830.

[3] R. Calaga, Crab cavities for the LHC upgrade, in Proceedings of the LHC Performance Workshop (CERN, Chamonix, France, 2012).

[4] B. Xiao, L. Alberty, S. Belomestnykh, I. Ben-Zvi et al., Design, prototyping, and testing of a compact superconducting double quarter wave crab cavity, Phys. Rev. ST Accel. Beams 18, 041004 (2015).

[5] S. Verdú-Andrés, K. Artoos, S. Belomestnykh, I. Ben-Zvi et al., Design and vertical tests of double-quarter wave cavity prototypes for the high-luminosity LHC crab cavity system, Phys. Rev. Accel. Beams 21, 082002 (2018).

[6] C. Zanoni, A. A. Carvalho, K. Artoos, S. Atieh et al., The crab cavities cryomodule for SPS test, J. Phys. Conf. Ser. 874, 012092 (2017).

[7] S. U. De Silva and J. R. Delayen, Design evolution and properties of superconducting parallel-bar rf-dipole deflecting and crabbing cavities, Phys. Rev. ST Accel. Beams 16, 012004 (2013).

[8] B. Hall, G. Burt, R. Apsimon, C. J. Lingwood et al., Design and testing of a four rod crab cavity for High Luminosity LHC, Phys. Rev. Accel. Beams 20, 012001 (2017).

[9] A. Grudiev, S. Atieh, R. Calaga, S. Calatroni et al., in Proceedings of SRF2015 (JACoW, Whistler, British Columbia, Canada, 2015), pp. 1205-1209.
[10] M. Benedikt, M. Capeans Garrido, F. Cerutti, B. Goddard et al., Future Circular Collider, CERN Technical Report No. CERN-ACC-2018-0058, 2018.

[11] S. Calatroni, 20 Years of experience with the $\mathrm{Nb} / \mathrm{Cu}$ technology for superconducting cavities and perspectives for future developments, Physica C (Amsterdam) 441, 95 (2006).

[12] A. Lunin, I. Gonin, M. Awida, T. Khabiboulline, V. Yakovlev, and A. Zholents, Design of a quasi-waveguide multicell deflecting cavity for the advanced photon source, Phys. Procedia 79, 54 (2015).

[13] COMSOL Multiphysics Ver. 5.3, COMSOL Multiphysics GmbH, Stockholm, Sweden, 2017.

[14] R. Collin, Foundations for microwave engineering, IEEE Press Series on Electromagnetic Wave Theory (Wiley, New York, 2001).

[15] CST-Computer Simulation Technology Ver. 2016, CST AG, Darmstadt, Germany, 2016.

[16] ANSYS HFSS—High Frequency Structural Simulator Ver. 2016 (ANSYS Inc., Canonsburg, Pennsylvania, USA, 2016).

[17] Computer aided three-dimensional interactive application Ver. R2015x, (Dassault Systèmes, Vélizy-Villacoublay, France, 2015).

[18] W. Venturini Delsolaro, Thin film research: Cern experience and possible future applications, Paper presented at the TTC meeting at INFN, Milano, Italy, 2018.

[19] A. Wolski, Beam Dynamics in High Energy Particle Accelerators (Imperial College Press, London, 2014), pp. 26-32.

[20] J. Barranco García, R. De Maria, A. Grudiev, R. Tomás García et al., Long term dynamics of the high luminosity Large Hadron Collider with crab cavities, Phys. Rev. Accel. Beams 19, 101003 (2016).

[21] P. Baudrenghien, K. Brodzinski, R. Calaga, O. Capatina et al., Functional Specifications of the LHC Prototype Crab Cavity System, Report No. CERN-ACC-NOTE-2013-003, 2013, https://cds.cern.ch/record/1520896.

[22] S. De Silva, J. Delayen, Z. Li, and H. Park, Proceedings of International Conference on RF Superconductivity (SRF'17), Lanzhou, China, 2017 (JACoW, Geneva, Switzerland, 2018), TUPB054, pp. 509-511.

[23] A. Gurevich, Multiscale mechanisms of SRF breakdown, Physica C (Amsterdam) 441, 38 (2006).

[24] V. Palmieri and R. Vaglio, Thermal contact resistance at the $\mathrm{Nb} / \mathrm{Cu}$ interface as a limiting factor for sputtered thin film RF superconducting cavities, Supercond. Sci. Technol. 29, 015004 (2016).

[25] P. Bauer, N. Solyak, G. Ciovati, G. Eremeev et al., Evidence for non-linear BCS resistance in SRF cavities, Physica C (Amsterdam) 441, 51 (2006).

[26] S. Bauer, W. Diete, B. Griep, M. Peiniger et al., in Proceedings of the 9th Workshop on RF Superconductivity, Santa Fe, New Mexico, USA, 1999, pp. 437-439.

[27] D. C. Mattis and J. Bardeen, Theory of the anomalous skin effect in normal and superconducting metals, Phys. Rev. 111, 412 (1958).

[28] T. Junginger, Investigations of the surface resistance of superconducting materials, Ph.D. thesis, Heidelberg U., 2012. 
[29] D. Longuevergne, F. Chatelet, G. Michel, F. R. G. Olry et al., in Proceedings of SRF2015 (JACoW, Whistler, British Columbia, Canada, 2015), pp. 923-929.

[30] F. Bernardini, J. Mittleman, H. Rushmeier, C. Silva et al., The ball-pivoting algorithm for surface reconstruction, IEEE Trans. Visual. Comput. Graph. 5, 349 (1999).

[31] P. Zhang, A. D'Elia, W. V. Delsolaro, and K. Artoos, Frequency pre-tuning of the niobium-sputtered quarterwave resonator for HIE-ISOLDE project at CERN, Nucl. Instrum. Methods Phys. Res., Sect. A 797, 101 (2015).

[32] F. Ruggiero, Single-beam collective effects in the LHC, CERN Technical Report No. CERN-SL-95-09-AP, 1995.

[33] J. Mitchell, G. Burt, N. Shipman, R. Calaga et al., in PreRelease Proc. SRF2017 (JACoW, Lanzhou, China, 2017).

[34] J. Tückmantel, Cavity-beam-transmitter interaction formula collection with derivation, CERN Technical Report No. CERN-ATS-Note-2011-002 TECH, 2010.

[35] F. Gerigk, S. Atieh, I. Aviles Santillana, W. Bartmann et al., Conceptual Design of the Low-Power and High-Power SPL: A Superconducting $\mathrm{H}^{-}$Linac at CERN (CERN, Geneva, 2014).

[36] S. Verdú-Andrés, S. Belomestnykh, I. Ben-Zvi, J. Skaritka et al., in Proceedings of IPAC2015 (JACoW, Richmond, VA, USA, 2015).

[37] W. Henneberg, R. Orthuber, and E. Steudel, Zur Wirkungsweise des Elektronenvervielfachers, Z. Tech. Phys. 17, 115 (1936).

[38] G. Burt and A. C. Dexter, Prediction of multipactor in the iris region of rf deflecting mode cavities, Phys. Rev. ST Accel. Beams 14, 122002 (2011).
[39] K. Ko and A. Candel, in Proceedings of the 25th International Linear Accelerator Conference, LINAC2010, Tsukuba, Japan (KEK, Tsukuba, Japan, 2010), pp. 1028-1032.

[40] G. Romanov, Stochastic features of multipactor in coaxial waveguides for travelling and standing waves, FermiLab Technical Report No. FERMILAB-PUB-11-003-TD, 2011.

[41] P. Zhang and W. V. Delsolaro, in Proceedings of SRF2015 (JACoW, Whistler, British Columbia, Canada, 2015), pp. 760-764.

[42] A. V. Gaponov and M. A. Miller, J. Exp. Theor. Phys. 34, 242 (1958)

[43] S. U. De Silva and J.R. Delayen, Cryogenic test of a proof-of-principle superconducting rf-dipole deflecting and crabbing cavity, Phys. Rev. ST Accel. Beams 16, 082001 (2013).

[44] ANSYS-ANalysis SYStem Ver. 17.2 (ANSYS Inc., Canonsburg, Pennsylvania, USA, 2016).

[45] ANSYS APDL-Parametric Design Language Ver. 17.2 (ANSYS Inc., Canonsburg, Pennsylvania, USA, 2016).

[46] X. Zhang, Z. Li, P. Zhang, H. Lin et al., in Pre-Release Proceedings of SRF2017 (JACoW, Lanzhou, China, 2017).

[47] T. Rubin, H. W. Altman, and H. L. Johnston, Coefficients of thermal expansion of solids at low temperatures. I. The thermal expansion of copper from 15 to $300^{\circ} \mathrm{K}$, J. Am. Chem. Soc. 76, 5289 (1954).

[48] M. Fink, T. Fabing, M. Scheerer, E. Semerad, and B. Dunn, Measurement of mechanical properties of electronic materials at temperatures down to $4.2 \mathrm{~K}$, Cryogenics $\mathbf{4 8}$, 497 (2008). 